\title{
On Vibrational Stabilizability of Nonlinear Systems ${ }^{1}$
}

\author{
R. BELLMAN, ${ }^{2}$ J. BENTSMAN, ${ }^{3}$ AND S. M. MEERKov ${ }^{4}$ \\ Dedicated to G. Leitmann
}

\begin{abstract}
Conditions of vibrational stabilizability for trivial solutions of nonlinear systems are derived. Several examples based on the classical equations of the theory of oscillations are given.
\end{abstract}

Key Words. Vibrational stabilizability, linear multiplicative vibrations, periodic forcing, Duffing equation, Van der Pol equation, Rayleigh equation, optimal shape of vibrations.

\section{Introduction}

Is it possible to stabilize an equilibrium point of a nonlinear finitedimensional system by zero average oscillations of its parameters? This is a question addressed in the present paper. A similar question regarding linear systems was discussed in Ref. 1 . It was shown that, if $A$ is a constant, nonderogatory matrix, the necessary and sufficient condition of vibrational stabilizability of $d x / d t=A x$ by zero average oscillations introduced in the elements of $A$ is $\operatorname{Tr}(A)<0$. Obviously, the stabilization of $d x / d t=A x$ by any type of additive zero average oscillations is impossible. Both of these statements are not true, in general, for nonlinear systems. References 2 and 3 gave examples of vibrational stabilizability when the linearized system had $\operatorname{Tr}(A)>0$. Moreover, as it was shown in Ref. 3, even additive vibrations might cause vibrational stabilizability in a specific type of nonlinear systems. These examples indicate that there are important differences between the phenomena of vibrational stabilizability in linear and nonlinear situations.

\footnotetext{
${ }^{1}$ This work was performed under the auspices of the US Department of Energy under Contract No. DE-ACO2-80ER10709.

${ }^{2}$ Professor, Departments of Mathematics, Electrical Engineering, and Medicine, University of Southern California, Los Angeles, California.

${ }^{3}$ Lecturer, Department of Electrical Engineering and Computer Science, University of Michigan, Ann Arbor, Michigan.

${ }^{4}$ Professor, Department of Electrical Engineering and Computer Science, University of Michigan, Ann Arbor, Michigan.
} 
The goal of this paper is to clarify some of these differences and to give conditions of vibrational stabilizability for several classes of nonlinear systems.

\section{The Problem}

Consider a system of the form

$$
d x / d t=X(x, \lambda), \quad X: R^{n} \times R^{m} \rightarrow R^{n},
$$

where $x \in R^{n}$ is the state, $t$ is dimensionless time, and $\lambda \in R^{m}$ is a parameter. Assume that (1) has an equilibrium point $x=x_{s}$ if $\lambda=\lambda_{0}=$ const.

Introduce vibrations in $\lambda$ according to the law

$$
\lambda=\lambda_{0}+a f(t / \epsilon),
$$

where $\lambda_{0}=$ const, $f(t / \epsilon)$ is a periodic vector-valued function with zero average, and $\epsilon, 0<\epsilon \ll 1$, and $a$ are scalars. For technical reasons, we assume that

$$
a=\alpha / \epsilon, \quad \alpha=\text { const. }
$$

This does not necessarily imply that the amplitude of oscillations is large. Indeed, for any given $\epsilon, \alpha$ can be chosen in such a manner that $a$ is not a large number. However, $\alpha$ is not an asymptotic parameter, whereas $\epsilon$ is. Thus, we consider oscillations of the form

$$
\lambda=\lambda_{0}+(\alpha / \epsilon) f(t / \epsilon) .
$$

Definition 2.1. An equilibrium point $x_{s}$ of (1), with $\lambda=\lambda_{0}$, is said to be vibrationally stabilizable if, for every $\delta>0$, there exists $0<\epsilon_{0} \ll 1$ such that (1), with $\lambda$ as in (2) and $0<\epsilon \leq \epsilon_{0}$, has an asymptotically stable periodic solution $x^{*}(t),-\infty<t<\infty$, characterized by

$$
\left\|\bar{x}^{*}(t)-x_{s}\right\|<\delta
$$

where

$$
\bar{x}^{*}(t)=\lim _{T \rightarrow \infty}(1 / T) \int_{0}^{T} x^{*}(t) d t .
$$

Thus, if $x_{s}$ is vibrationally stabilizable, there exists a zero average oscillation of $\lambda$ which causes a bifurcation of this equilibrium point into an asymptotically stable periodic solution $x^{*}(t)$, the average value of which is arbitrarily close to $x_{\mathrm{s}}$. 
Assume that (1), with $\lambda$ as in (2), has the form

$$
d x / d t=X\left(x, \lambda_{0}\right)+(\alpha / \epsilon) X_{1}(t / \epsilon, x), \quad X_{1}: R_{+} \times R^{n} \rightarrow R^{n},
$$

where $X_{1}(t / \epsilon, \cdot)$ is a periodic function. Equation (5) describes a large class of nonlinear systems with vibrations. Indeed, several examples of $X_{1}(t / \epsilon, x)$ can be given as follows.

(i) $X_{1}(t / \epsilon, x)=L(t / \epsilon)$, where $L(t / \epsilon)$ is a periodic average zero vector. In this case, the vibrations will be referred to as vector additive. In a specific case when all but the last components of $L(t / \epsilon)$ are zero, the vibrations will be referred to as periodic forcing.

(ii) $X_{1}(t / \epsilon, x)=B(t / \epsilon) x$, where $B(t / \epsilon)$ is a zero average periodic matrix. In this case, the vibrations will be called linear multiplicative.

(iii) $X_{1}(t / \epsilon, x)=B(t / \epsilon) \Gamma(x), \Gamma: R^{n} \rightarrow R^{n}$. Here, the vibrations are nonlinear multiplicative.

In what follows, conditions of vibrational stabilizability of trivial solutions for nonlinear systems by linear multiplicative and vector additive vibrations as well as by periodic forcing are derived. In Section 3, the method of analysis is described. In Sections 4-6, conditions of vibrational stabilizability are formulated and corresponding examples are given. In Section 7 , the optimal shape of vibrations is discussed.

\section{The Method}

In the fast time $\tau=t / \epsilon$, Eq. (5) can be rewritten as

$$
d x / d \tau=\epsilon X(x)+\alpha X_{1}(\tau, x), \quad X(x) \equiv X\left(x, \lambda_{0}\right) .
$$

Introduce an equation

$$
d x / d \tau=\alpha X_{1}(\tau, x),
$$

and assume that there exists a unique solution of (7) defined by every initial condition $x_{0} \in \Omega \subset R^{n}$; for all $\tau \geq 0$. Denote the general solution of (7) as

$$
x(\tau)=h(\tau, c), \quad c=\text { const. }
$$

Introduce in (6) a substitution,

$$
x(\tau)=h(\tau, y(\tau)), \quad y \in R^{n} .
$$

Assuming that $X_{1}(\tau, x)$ is differentiable with respect to $x$, for all $\tau \geq 0$, and recognizing that

$$
\operatorname{det}[\partial h(\tau, y) / \partial y]=\operatorname{det}[\partial h(0, y) / \partial y] \exp \left\{\int_{0}^{\tau} \operatorname{Tr}\left[\partial X_{1}(\tau, y) / \partial y\right] d \tau\right\},
$$


we obtain the following equation for $y(\tau)$ :

$$
d y / d \tau=\epsilon[\partial h / \partial y]^{-1} X(h(\tau, y)) \triangleq \epsilon Y(\tau, y) .
$$

Introduce, finally, the averaged equation

$$
d z / d \tau=\epsilon Z(z),
$$

where

$$
Z(y)=\lim _{T \rightarrow \infty}(1 / T) \int_{0}^{T}[\partial h / \partial y]^{-1} X(h(\tau, y)) d \tau .
$$

Lemma 3.1. Assume that:

(a) $h(\tau, c)$ is periodic with respect to $\tau$;

(b) $\|Y(\tau, y)\| \leq N, \forall \tau \in[0, \infty), \forall y \in \Omega \subset R^{n}$,

$$
\left\|Y\left(\tau, y^{\prime}\right)-Y\left(\tau, y^{\prime \prime}\right)\right\| \leq K\left\|y^{\prime}-y^{\prime \prime}\right\|, \forall \tau \in[0, \infty), \forall y \in \Omega \subset R^{n} ;
$$

(c) $Z(y)$ is continuously differentiable for all $y \in \Omega$. Then,

(i) the equilibrium point $x_{s}$ of (1), with $\lambda=\lambda_{0}$, is vibrationally stabilizable if there exists an equilibrium point $z_{s}$ of $(10)$ such that

$$
\begin{aligned}
& \lim _{T \rightarrow \infty}(1 / T) \int_{0}^{T} h\left(\tau, z_{s}\right)=x_{s}, \\
& Q=[\partial Z / \partial z]_{z=z_{s}} \text { is a Hurwitz matrix; }
\end{aligned}
$$

(ii) the equilibrium point $x_{s}$ of (1) with $\lambda=\lambda_{0}$ is not vibrationally stabilizable if either condition (12) is not met or $Q$ is an unstable matrix having no pure imaginary eigenvalues.

Proof. Under the Assumptions (a)-(c) of Lemma 3.1, the conditions of the second theorem of the averaging principle (Ref. 4, p. 497) are satisfied. Thus, for every $\eta>0$, there exists $\epsilon_{0}(\eta)$ such that (9), with $0<\epsilon \leq \epsilon_{0}$, has a unique periodic solution $y^{*}(\tau)$ characterized by

$$
\left\|y^{*}(\tau)-z_{s}\right\|<\eta
$$

where $z_{s}$ is an equilibrium position of $(10) ; y^{*}(\tau)$ is asymptotically stable if $Q$ is Hurwitz; $y^{*}(\tau)$ is unstable if $Q$ is an unstable matrix without imaginary eigenvalues; the period of $y^{*}(\tau)$ is equal to the period of $h(\tau, \cdot)$.

Now, since $h(\tau, c)$ is a solution of (7) defined for all $\tau \in[0, \infty)$, there exists a Lipschitz constant $M$ such that

$$
\left\|h\left(\tau, y^{*}(\tau)\right)-h\left(\tau, z_{s}\right)\right\| \leq M\left\|y^{*}(\tau)-z_{s}\right\|, \quad \forall \tau \in[0, \infty), \forall y^{*}, z_{s} \in \Omega .
$$


Consequently, choosing $\eta$ in Eq. (14) as $\delta / M$, we obtain

$$
\begin{aligned}
& \left\|\lim _{T \rightarrow \infty}(1 / T) \int_{0}^{T} h\left(\tau, y^{*}(\tau)\right) d \tau-\lim _{\tau \rightarrow \infty}(1 / T) \int_{0}^{T} h\left(\tau, z_{s}\right) d \tau\right\| \\
& \quad \leq \lim _{T \rightarrow \infty}(M / T) \int_{0}^{T}\left\|y^{*}(\tau)-z_{s}\right\| d \tau \leq \delta .
\end{aligned}
$$

If condition (12) takes place, from condition (15) it then follows that

$$
\lim _{T \rightarrow \infty}(1 / T) \int_{0}^{T} h\left(\tau, y^{*}(\tau)\right) d \tau-x_{s} \|<\delta .
$$

Due to Assumption (a) of Lemma 3.1 and due to $(8), h\left(\tau, y^{*}(\tau)\right)$ is a periodic solution of $(6)$,

$$
x^{*}(\tau)=h\left(\tau, y^{*}(\tau)\right) \text {. }
$$

This means that condition (16) coincides with Ineq. (3). Thus, under Assumptions (a)-(c), vibrational stabilizability of $x_{s}$ takes place if $z_{s}$ is asymptotically stable. This proves proposition (i) of Lemma 3.1. The equilibrium point $x_{s}$ is not vibrationally stabilizable if either no $z_{s}$ satisfy condition (12) or $Q$ is an unstable matrix having no eigenvalues with zero real part. This proves proposition (ii).

Lemma 3.1 constitutes the basis for the investigation of conditions of vibrational stabilizability. Theorems $4.1-4.3$ below are proved by reducing the problem at hand to Lemma 3.1. These reductions do not present serious difficulties; therefore, the proofs are not included in the paper.

\section{Linear Multiplicative Vibrations}

This case constitutes a bridge between the linear and nonlinear situations. Namely, only in this case do the conditions of vibrational stabilizability for linear and nonlinear systems coincide.

Theorem 4.1. Assume that $X\left(x, \lambda_{0}\right)$ is analytic around $x=0$, $X\left(0, \lambda_{0}\right)=0$, and there exists $\Omega \subset R^{n}(0 \in \Omega)$ such that

$$
\begin{aligned}
& \left\|X\left(x, \lambda_{0}\right)\right\| \leq N, \quad \forall x \in \Omega, \\
& \left\|X\left(x^{\prime}, \lambda_{0}\right)-X\left(x^{\prime \prime}, \lambda_{0}\right)\right\| \leq K\left(\lambda_{0}\right)\left\|x^{\prime}-x^{\prime \prime}\right\|, \quad \forall x^{\prime}, x^{\prime \prime} \in \Omega .
\end{aligned}
$$

Assume also that the state transition matrix $\Phi(\tau, 0), \tau \in[0, \infty)$, of the equation $d x / d \tau=\alpha B(\tau) x$ is periodic. Let $d x / d t=A x$ be the linearization of Eq. (1) around zero, where $A=\left[\partial X\left(x, \lambda_{0}\right) / \partial x\right]_{x=0}$ is a nonderogatory 
matrix. Then,

(i) 0 of Eq. (1), with $\lambda=\lambda_{0}$, is vibrationally stabilizable by linear multiplicative vibrations if $\operatorname{Tr}(A)<0$;

(ii) 0 of Eq. (1), with $\lambda=\lambda_{0}$, is not vibrationally stabilizable by linear multiplicative vibrations if $\operatorname{Tr}(A)>0$.

Example 4.1. Consider the Duffing equation,

$\ddot{x}+a \dot{x}-b x+c x^{3}=0, \quad a, b, c>0$.

The linearization around $x_{s}=0$ is

$\ddot{x}+a \dot{x}-b x=0$.

Since $a>0, \operatorname{Tr}(A)$ is negative. Therefore, 0 is vibrationally stabilizable by linear multiplicative vibrations.

Example 4.2. Consider the Ray'eigh equation, $\ddot{x}+\mu\left(\dot{x}^{2} / 3-1\right) \dot{x}+x=0, \quad \mu>0$.

Obviously, here $\operatorname{Tr}(A)>0$. Hence, 0 is not vibrationally stabilizable by linear multiplicative vibrations.

\section{Periodic Forcing}

In this section, we consider a system of the form

$$
x^{(n)}+a_{1} x^{(n-1)}+\cdots+a_{n} x+f\left(x, \ldots, x^{(n-1)}\right)=0,
$$

where $x$ is a scalar, $x^{(i)}$ is the $i$ th time derivative of $x, a_{i}$ are constant coefficients, and $f\left(x, \ldots, x^{(n-1)}\right)$ is a polynomial such that

$$
f(0, \ldots, 0)=0, \quad \operatorname{grad} f(0, \ldots, 0)=0 .
$$

Adding a zero average periodic forcing $(\alpha / \epsilon) l(t / \epsilon)$, we rewrite (17) as

$$
x^{(n)}+a_{1} x^{(n-1)}+\cdots+a_{n} x+f\left(x_{1}, \ldots, x^{(n-1)}\right)=(\alpha / \epsilon) l(t / \epsilon) .
$$

Lemma 5.1. An unstable 0 of Eq. (17) is vibrationally stabilizable by periodic forcing only if $f\left(x, \ldots, x^{(n-1)}\right)$ has terms of the form

$$
x^{(n-i)}\left[x^{(n-1)}\right]^{2 k}, \quad k=1,2, \ldots, \quad i=1, \ldots, n .
$$

Theorem 5.1. Assume that $a_{i}, i=1, \ldots, n$, in Eq. (17) are such that $a_{i}>0, \quad i \neq s, \quad a_{s}=k<0$. 
Assume also that there exists $c_{s}^{*}=$ const such that Eq. (17), with $a_{s}^{\prime}=k+c_{s}^{*}$, has asymptotically stable trivial solution. Then, 0 of Eq. (17) is vibrationally stabilizable by periodic forcing if

$$
f\left(x, \ldots, x^{(n-1)}\right)=\mu x^{(n-s)}\left[x^{(n-1)}\right]^{2 k}, \quad k=1,2, \ldots, \quad \mu>0 .
$$

Example 5.1. Consider the Rayleigh equation,

$$
\ddot{x}+\mu\left(\dot{x}^{2} / 3-1\right) \dot{x}+x=0, \quad \mu>0 .
$$

Here,

$$
f(x, \dot{x})=(\mu / 3) \dot{x}(\dot{x})^{2}, \quad a_{s}=-\mu, c_{s}^{*}>\mu .
$$

Thus, as it follows from Theorem 5.1, 0 is vibrationally stabilizable by periodic forcing.

Example 5.2. Consider the Van der Pol equation,

$$
\ddot{x}+\mu\left(x^{2}-1\right) \dot{x}+x=0 .
$$

Here,

$$
f(x, \dot{x})=\mu x^{2} \dot{x} .
$$

Obviously, the necessary condition of Lemma 5.1 is not met. Thus, 0 is not vibrationally stabilizable by periodic forcing.

\section{Vector Additive Vibrations}

To formulate a condition for vibrational stabilizability by vector additive vibrations, consider the Taylor expansion of $X(x) \equiv X\left(x, \lambda_{0}\right)$ around $x=x^{*}$,

$$
\left.X(x)=X\left(x^{*}\right)+\sum_{i=1}^{\infty}(1 / i !)\{\partial X / \partial x]_{x=x^{*}}\left(x-x^{*}\right)\right\}^{i^{\otimes}},
$$

where

$$
\left[\left(v_{1}, \ldots, v_{n}\right)\right]^{{ }^{\otimes}}=\left[v_{1}^{r}, \ldots, v_{n}^{r}\right]^{\prime} .
$$

We will say that $X(x)$ is an odd r-algebraic function in the vicinity of $x^{*}$, if:

(i) the expansion (18) has $r<\infty$ terms;

(ii) the expansion (18) has no terms with $i=2 k, k=0,1, \ldots, r / 2+1$. 
The $i$ th term of Eq. (18), with $x^{*}=0$ and $x=y+u$, can be represented as

$$
(1 / i !)\left\{[\partial X / \partial x]_{x=0}(y+u)\right\}^{i^{\infty}}=(1 / i !) v_{i}+[1 /(i-1) !] P_{i} y+\text { h.o.t. }(y),
$$

where the elements of the vector $v_{i}$ are algebraic forms of order $i$ with respect to the components of vector $u$ and the elements of the matrix $P_{i}$ are algebraic forms of order $i-1$ with respect to $u$. The element $p_{l m}$ of the matrix $P_{3}$, for instance, is

$$
\begin{array}{r}
p_{l m}=d_{l m}\left[\left(d_{l 1} u_{1}+\cdots+d_{l n} u_{n}\right)^{2}-\sum_{j=1}^{n} \sum_{i=1}^{n} d_{l i} u_{i} d_{l j} u_{j}\right], \\
l, m=1, \ldots, n, \quad i \neq j, \quad i \neq m, \quad j \neq m,
\end{array}
$$

where

$$
d_{l m}=d_{l m}\{X\}=\partial X_{l} / \partial x_{m}, d_{l m} d_{l k}=d_{i m} d_{l k}\{X\}=\partial^{2} X_{l} / \partial x_{m} \partial x_{k} .
$$

Define $u$ in Eq. (19) via vector additive vibrations $\alpha L(\tau)$ as

$$
u(\tau) \triangleq \alpha \int L(\tau) d \tau
$$

and introduce a matrix

$$
\begin{aligned}
\bar{A} \stackrel{\Delta}{\triangleq}[\partial X / \partial x]_{x=0}+\lim _{\tau \rightarrow \infty}(1 / T) \int_{0}^{T}\left[(1 / 2 !) P_{3}(u(\tau))+(1 / 4 !) P_{5}(u(\tau))\right. \\
\\
\left.+\cdots+(1 /(S-1) !) P_{S}(u(\tau))\right] d \tau
\end{aligned}
$$

where $S=r-1$, if $r$ is even, and $S=r$, if $r$ is odd.

Theorem 6.1. Assume that $X\left(x, \lambda_{0}\right)$ in the vicinity of 0 is an odd $r$-algebraic function. Then,

(i) 0 of Eq. (1), with $\lambda=\lambda_{0}$, is vibrationally stabilizable by vector additive vibrations if there exists $u(\tau)$ such that $\bar{A}$ is a Hurwitz matrix;

(ii) 0 of Eq. (1), with $\lambda=\lambda_{0}$, is not vibrationally stabilizable if, for any $u(\tau), \bar{A}$ is an unstable matrix, provided $\bar{A}$ has no imaginary eigenvalues.

Example 6.1. Consider the Van der Pol equation,

$$
\left[\begin{array}{c}
\dot{x}_{1} \\
\dot{x}_{2}
\end{array}\right]=\left[\begin{array}{c}
x_{2} \\
-x_{1}+\mu x_{2}-\mu x_{1}^{2} x_{2}
\end{array}\right], \quad \mu>0
$$


Introduce vector additive vibrations in Eq. (23), in the form

$$
\left[\begin{array}{c}
\dot{x}_{1} \\
\dot{x}_{2}
\end{array}\right]=\left[\begin{array}{c}
x_{2} \\
-x_{1}+\mu x_{2}-\mu x_{1}^{2} x_{2}
\end{array}\right]+(\alpha / \epsilon)\left[\begin{array}{l}
l(t / \epsilon) \\
l(t / \epsilon)
\end{array}\right] .
$$

Obviously, the right-hand side of Eq. (23) in the vicinity of $x_{1}=0, x_{2}=0$ is an odd 3-algebraic function. Thus, the property of vibrational stabilizability of Eq. (23) is determined by the matrix $\bar{A}$. In the case of Eq. (24), the matrix $\bar{A}$ is

$$
\bar{A}=\left[\begin{array}{cc}
0 & 1 \\
-\left(1+2 \mu \overline{u^{2}(\tau)}\right) & \mu\left(1-\overline{u^{2}(\tau)}\right)
\end{array}\right],
$$

where

$$
\overline{u^{2}(\tau)}=\lim _{T \rightarrow \infty}(1 / T) \int_{0}^{T} u^{2}(\tau) d \tau
$$

Consequently, 0 of the Van der Pol equation is vibrationally stabilizable by vector additive vibrations if $\overline{u^{2}(\tau)}>1$.

\section{Optimal Shape of Vibrations}

Normally, the amplitudes of the stabilizing vibrations are bounded; i.e., the zero average oscillations in Eq. (2) must satisfy the inequalities

$$
N \leq a f(\tau) \leq M,
$$

where $M$ and $N$ are $m$-vectors with positive and negative components, respectively. Consequently, it is important to find the optimal (from the point of view of ensuring the stability property of equilibrium positions) shape of vibrations. In the case of linear systems, the optimal shape of vibrations was found in Ref. 1 to be a rectangular waveform. This conclusion holds true for the nonlinear systems considered in this paper as well. Indeed, assume that

$$
f_{i}(\tau)=\sum k_{i}^{s} \sin \left(s \tau+\phi_{i}^{s}\right)
$$

Then, for $u_{i}(\tau)$ as defined in Eq. (21), we obtain

$$
\overline{u_{i}^{2}(\tau)}=\left(\alpha^{2} / 2\right) \sum\left[\left(k_{i}^{s}\right)^{2} / \omega^{2} s^{2}\right] \text {. }
$$

Since, as it follows from Eqs. (21)-(24), the effect of vibrational control is proportional to $\overline{u^{2}(\tau)}$, and since the effect of higher harmonics in $\overline{u^{2}(\tau)}$ is decreasing as $1 / s^{3}$, we conclude that the optimal shape of vibrations is the one which maximizes the amplitude of the fundamental frequency. Thus, 
we obtain the following constrained dynamic optimization problem (Ref. $5)$ : Find a piecewise continuous $2 \pi$-periodic function $f_{i}^{*}(\tau)$ which maximizes

$$
\int_{0}^{2 \pi} f_{i}(\tau) \cos (\tau+\phi) d \tau
$$

subject to the constraints

$$
\int_{0}^{2 \pi} f_{i}(\tau) d \tau=0, \quad N_{i} \leq f_{i}(\tau) \leq M_{i}
$$

The solution of this problem, based on the methods of Ref. 5, can be stated in the following lemma.

Lemma 7.1. The optimal shape of vibrations is a rectangular waveform.

\section{Conclusions}

Thus, in the case of linear multiplicative vibrations, the conditions of vibrational stabilizability depend only on the properties of the linear part of the system (Theorem 4.1). In the case of periodic forcing, they depend only on the nonlinearity (Theorem 5.1). In the case of vector additive vibrations, they depend on both (Theorem 6.1). And, if one happens to tremble, there is a way to do it optimally (Lemma 7.1).

\section{References}

1. MeErkov, S. M., Principle of Vibrational Control: Theory and Applications, IEEE Transactions on Automatic Control, Vol. AC-25, pp. 755-762, 1980.

2. MEerkov, S. M., Condition of Vibrational Stabilizability for a Class of Nonlinear Systems, IEEE Transactions on Automatic Control, Vol. AC-27, pp. 485-487, 1982.

3. Bellman, R., Bentsman, J., and Meerkov, S. M., Vibrational Control of Systems with Arrhenius Dynamics, Journal of Mathematical Analysis and Applications, Vol. 91, pp. 152-191, 1983.

4. Bogoliubov, N. N., and Mitropolsky, Yu. A., Asymptotic Methods in the Theory of Nonlinear Oscillations, Gordon and Breach, New York, New York, 1961.

5. Leitmann, G., The Calculus of Variations and Optimal Control, Plenum Press, New York, New York, 1981. 